\title{
Recurrence and differential relations for spherical spinors
}

\author{
Radosław Szmytkowski
}

\author{
Atomic Physics Division, Department of Atomic Physics and Luminescence, \\ Faculty of Applied Physics and Mathematics, Gdańsk University of Technology, \\ Narutowicza 11/12, PL 80-233 Gdańsk, Poland \\ email: radek@mif.pg.gda.pl
}

Published as: J. Math. Chem. 42 (2007) 397-413

doi: $10.1007 / \mathrm{s} 10910-006-9110-0$

\begin{abstract}
We present a comprehensive table of recurrence and differential relations obeyed by spin one-half spherical spinors (spinor spherical harmonics) $\Omega_{\kappa \mu}(\mathbf{n})$ used in relativistic atomic, molecular, and solid state physics, as well as in relativistic quantum chemistry. First, we list finite expansions in the spherical spinor basis of the expressions $\mathbf{A} \cdot \mathbf{B} \Omega_{\kappa \mu}(\mathbf{n})$ and $\mathbf{A} \cdot(\mathbf{B} \times \mathbf{C}) \Omega_{\kappa \mu}(\mathbf{n})$, where $\mathbf{A}, \mathbf{B}$, and $\mathbf{C}$ are either of the following vectors or vector operators: $\mathbf{n}=\mathbf{r} / r$ (the radial unit vector), $\mathbf{e}_{0}, \mathbf{e}_{ \pm 1}$ (the spherical, or cyclic, versors), $\boldsymbol{\sigma}$ (the $2 \times 2$ Pauli matrix vector), $\hat{\mathbf{L}}=-i \mathbf{r} \times \nabla I$ (the dimensionless orbital angular momentum operator; $I$ is the $2 \times 2$ unit matrix), $\hat{\mathbf{J}}=\hat{\mathbf{L}}+\frac{1}{2} \boldsymbol{\sigma}$ (the dimensionless total angular momentum operator). Then, we list finite expansions in the spherical spinor basis of the expressions $\mathbf{A} \cdot \mathbf{B} F(r) \Omega_{\kappa \mu}(\mathbf{n})$ and $\mathbf{A} \cdot(\mathbf{B} \times \mathbf{C}) F(r) \Omega_{\kappa \mu}(\mathbf{n})$, where at least one of the objects $\mathbf{A}, \mathbf{B}, \mathbf{C}$ is the nabla operator $\boldsymbol{\nabla}$, while the remaining ones are chosen from the set $\mathbf{n}, \mathbf{e}_{0}, \mathbf{e}_{ \pm 1}, \boldsymbol{\sigma}, \hat{\mathbf{L}}, \hat{\mathbf{J}}$.

KEY WORDS: spherical spinors, spinor spherical harmonics, angular momentum, recurrence relations, differential relations
\end{abstract}

AMS subject classification: 33C50, 33C55, 33E30, 81Q99

\section{Introduction}

The spin one-half spherical spinors (spinor spherical harmonics) emerge in relativistic quantum mechanics in the context of the separation of spherical variables when solving the central-field Dirac problem, e.g., in the relativistic analysis of one-electron atoms [1 5]. In the relativistic theory of many-electron systems (including atoms, molecules, and the solid state), they enter elementary oneelectron Dirac central-field orbitals of which approximate multi-electron wave functions describing such systems are frequently constructed (see numerous references cited in Refs. 6 6]).

Despite the so well documented practical importance of the spherical spinors, thus far relatively little space has been devoted in the literature to systematic studies or presentations of their properties. Standard textbooks or reference works on the angular momentum theory, such as Refs. 10 13, discuss the spherical spinors only superficially. In fact, even in the most comprehensive relevant treatise by Varshalovich et al. 14 the spherical spinors have been treated much less exhaustively than scalar or vector spherical harmonics. In consequence, atomic and molecular researchers usually have to derive particular properties of the spherical spinors ad hoc, as exemplified by Refs. [15 [19]. As a part of our research program (in this connection, see also Ref. [20]) aimed at changing this unsatisfactory situation, in this paper we present a comprehensive table of recurrence and differential relations obeyed by the spherical spinors. 


\section{Preliminaries}

\subsection{Definitions}

Let $\mathbf{e}_{x}, \mathbf{e}_{y}, \mathbf{e}_{z}$ be versors of some right-handed Cartesian coordinate system. The cyclic versors $\mathbf{e}_{0}$, $\mathbf{e}_{ \pm 1}$, are defined through the relationships

$$
\mathbf{e}_{0}=\mathbf{e}_{z}, \quad \mathbf{e}_{ \pm 1}=\mp \frac{1}{\sqrt{2}}\left(\mathbf{e}_{x} \pm i \mathbf{e}_{y}\right)
$$

The dimensionless orbital $(\hat{\mathbf{L}})$ and total $(\hat{\mathbf{J}})$ angular momentum operators (both with respect to the center of the aforementioned Cartesian system) are defined as

$$
\hat{\mathbf{L}}=-i \mathbf{r} \times \nabla I
$$

and

$$
\hat{\mathbf{J}}=\hat{\mathbf{L}}+\frac{1}{2} \boldsymbol{\sigma},
$$

respectively. In Eqs. (2.1.2) and (2.1.3), and hereafter, $I$ is the $2 \times 2$ unit matrix, while $\sigma$ is the $2 \times 2$ Pauli matrix vector

$$
\boldsymbol{\sigma}=\sigma_{x} \mathbf{e}_{x}+\sigma_{y} \mathbf{e}_{y}+\sigma_{z} \mathbf{e}_{z},
$$

with

$$
\sigma_{x}=\left(\begin{array}{cc}
0 & 1 \\
1 & 0
\end{array}\right), \quad \sigma_{y}=\left(\begin{array}{cc}
0 & -i \\
i & 0
\end{array}\right), \quad \sigma_{z}=\left(\begin{array}{cc}
1 & 0 \\
0 & -1
\end{array}\right) .
$$

Let $\mathbf{n}=\mathbf{r} / r$ be a unit radius vector with respect to the origin of the aforementioned Cartesian system. The spatial orientation of $\mathbf{n}$ is uniquely determined by specifying two angles, $0 \leqslant \theta \leqslant \pi$ and $0 \leqslant \varphi<2 \pi$, such that

$$
\mathbf{e}_{x} \cdot \mathbf{n}=\sin \theta \cos \varphi, \quad \mathbf{e}_{y} \cdot \mathbf{n}=\sin \theta \sin \varphi, \quad \mathbf{e}_{z} \cdot \mathbf{n}=\cos \theta .
$$

Evidently, $\theta$ and $\varphi$ are, respectively, the polar and the azimuthal angles in the spherical system of coordinates, with its polar and azimuthal axes directed along the Cartesian versors $\mathbf{e}_{z}$ and $\mathbf{e}_{x}$, respectively.

We define the spin one-half spherical spinors, hereafter denoted as $\Omega_{\kappa \mu}(\mathbf{n})$, as two-component functions of the unit vector $\mathbf{n}$ (or, equivalently, of the aforementioned angles $\theta$ and $\varphi$ ) of the form

$$
\Omega_{\kappa \mu}(\mathbf{n})=\left(\begin{array}{c}
\operatorname{sgn}(-\kappa) \sqrt{\frac{\kappa+\frac{1}{2}-\mu}{2 \kappa+1}} Y_{l, \mu-1 / 2}(\mathbf{n}) \\
\sqrt{\frac{\kappa+\frac{1}{2}+\mu}{2 \kappa+1}} Y_{l, \mu+1 / 2}(\mathbf{n})
\end{array}\right),
$$

with $\kappa \in\{ \pm 1, \pm 2, \ldots\}, \mu \in\left\{-|\kappa|+\frac{1}{2},-|\kappa|+\frac{3}{2}, \ldots,|\kappa|-\frac{1}{2}\right\}$, and

$$
l=\left|\kappa+\frac{1}{2}\right|-\frac{1}{2}= \begin{cases}\kappa & \text { for } \kappa>0 \\ -\kappa-1 & \text { for } \kappa<0 .\end{cases}
$$

In Eq. (2.1.7),

$$
Y_{l m}(\mathbf{n})=\sqrt{\frac{2 l+1}{4 \pi} \frac{(l-m) !}{(l+m) !}} P_{l}^{(m)}(\cos \theta) \mathrm{e}^{i m \varphi}
$$

is the scalar spherical harmonics, with

$$
P_{l}^{(m)}(\xi)=\frac{(-)^{m}}{2^{l} l !}\left(1-\xi^{2}\right)^{m / 2} \frac{\mathrm{d}^{l+m}}{\mathrm{~d} \xi^{l+m}}\left(\xi^{2}-1\right)^{l} \quad(-1 \leqslant \xi \leqslant+1)
$$


being the associated Legendre function of the first kind. (The phases in Eqs. (2.1.9) and (2.1.10) have been chosen so that the spherical harmonics (2.1.9) conforms to the Condon-Shortley [21] phase convention; in this connection, see also the remark concluding Sec. 2.2.)

In this work, we shall label the spherical spinors with the two indices $\kappa$ and $\mu$. However, it should be mentioned that in the relevant literature one encounters also numerous examples of labeling these functions by three indices $j, l$, and $\mu$, with the first index in this triple related to $\kappa$ through

$$
j=|\kappa|-\frac{1}{2},
$$

with $l$ defined as in Eq. (2.1.8), and with $\mu$ assuming the same value as explained below Eq. (2.1.7). In view of the relations (2.1.8) and (2.1.11), and the converse one,

$$
\kappa=(l-j)(2 j+1),
$$

both labeling schemes are completely equivalent.

\subsection{Remarks}

Preparing the collection of formulas presented in Sec. 3 we have made an attempt to minimize the number of entries (with a few exceptions when the operator $\hat{\mathbf{J}}$ has been involved). To this end, we have made an extensive use of the property

$$
\mathbf{A} \cdot(\mathbf{B} \times \mathbf{C})=(\mathbf{A} \times \mathbf{B}) \cdot \mathbf{C},
$$

valid alike for ordinary vectors and vector operators. Also, we have extensively exploited the identities like

$$
\begin{aligned}
& \hat{\mathbf{L}} \times \hat{\mathbf{L}}=i \hat{\mathbf{L}}, \quad \boldsymbol{\sigma} \times \boldsymbol{\sigma}=2 i \boldsymbol{\sigma}, \quad \hat{\mathbf{J}} \times \hat{\mathbf{J}}=i \hat{\mathbf{J}}, \\
& \mathbf{n} \times \boldsymbol{\nabla}=-\boldsymbol{\nabla} \times \mathbf{n}, \quad \mathbf{n} \cdot \hat{\mathbf{L}}=\hat{\mathbf{L}} \cdot \mathbf{n}=0, \quad \boldsymbol{\nabla} \cdot \hat{\mathbf{L}}=\hat{\mathbf{L}} \cdot \boldsymbol{\nabla}=0,
\end{aligned}
$$

etc., to reduce operators acting on the spherical spinors to the simplest possible forms. If a result of such a reduction of a particular operator has been found to be a scalar multiple of the identity, the action of this operator on the spherical spinors has not been displayed in the table. For instance, the equation

$$
(\mathbf{n} \times \hat{\mathbf{L}}) \times \mathbf{n} \Omega_{\kappa \mu}(\mathbf{n})=2 i \Omega_{\kappa \mu}(\mathbf{n})
$$

has not been included in Sec. 3 since it reflects the operator identity

$$
\mathbf{n} \times(\hat{\mathbf{L}} \times \mathbf{n})=(\mathbf{n} \times \hat{\mathbf{L}}) \times \mathbf{n}=2 i I
$$

rather than some particular property of the spherical spinors.

In Sec. 3.3, $F(r)$ is a (once or twice, depending on the needs) differentiable, and otherwise arbitrary, function of the radial variable $r=|\mathbf{r}|$.

Before proceeding to the table, a word of caution is still in order. It appears that, analogously to the case of scalar spherical harmonics, recurrence and differential relations obeyed by the spherical spinors depend on the choices of phases in the defining equations. In other words, if the net phase of spherical spinors in use differs from that following from our Eqs. (2.1.7), (2.1.9), and (2.1.10), or if the Pauli matrix $\sigma_{y}$ is defined with the opposite sign, as it occasionally happens in the literature, signs in some of the relationships listed in Sec. 3 may need to be changed. 


\section{Table of recurrence and differential relations for spherical spinors}

\subsection{Algebraic recurrence relations}

$$
\begin{aligned}
& \mathbf{e}_{0} \cdot \mathbf{n} \Omega_{\kappa \mu}(\mathbf{n})=-\frac{2 \mu}{4 \kappa^{2}-1} \Omega_{-\kappa \mu}(\mathbf{n})+\frac{\sqrt{\left(\kappa+\frac{1}{2}\right)^{2}-\mu^{2}}}{|2 \kappa+1|} \Omega_{\kappa+1, \mu}(\mathbf{n}) \\
& +\frac{\sqrt{\left(\kappa-\frac{1}{2}\right)^{2}-\mu^{2}}}{|2 \kappa-1|} \Omega_{\kappa-1, \mu}(\mathbf{n}) \\
& \mathbf{e}_{ \pm 1} \cdot \mathbf{n} \Omega_{\kappa \mu}(\mathbf{n})= \pm \sqrt{2} \frac{\sqrt{\kappa^{2}-\left(\mu \pm \frac{1}{2}\right)^{2}}}{4 \kappa^{2}-1} \Omega_{-\kappa, \mu \pm 1}(\mathbf{n}) \\
& +\frac{\sqrt{\left(\kappa \pm \mu+\frac{1}{2}\right)\left(\kappa \pm \mu+\frac{3}{2}\right)}}{\sqrt{2}(2 \kappa+1)} \Omega_{\kappa+1, \mu \pm 1}(\mathbf{n}) \\
& -\frac{\sqrt{\left(\kappa \mp \mu-\frac{1}{2}\right)\left(\kappa \mp \mu-\frac{3}{2}\right)}}{\sqrt{2}(2 \kappa-1)} \Omega_{\kappa-1, \mu \pm 1}(\mathbf{n}) \\
& \mathbf{n} \cdot \boldsymbol{\sigma} \Omega_{\kappa \mu}(\mathbf{n})=-\Omega_{-\kappa \mu}(\mathbf{n}) \\
& \mathbf{e}_{0} \cdot \boldsymbol{\sigma} \Omega_{\kappa \mu}(\mathbf{n})=-\frac{2 \mu}{2 \kappa+1} \Omega_{\kappa \mu}(\mathbf{n})-2 \frac{\sqrt{\left(\kappa+\frac{1}{2}\right)^{2}-\mu^{2}}}{|2 \kappa+1|} \Omega_{-\kappa-1, \mu}(\mathbf{n}) \\
& \mathbf{e}_{ \pm 1} \cdot \boldsymbol{\sigma} \Omega_{\kappa \mu}(\mathbf{n})= \pm \sqrt{2} \frac{\sqrt{\kappa^{2}-\left(\mu \pm \frac{1}{2}\right)^{2}}}{2 \kappa+1} \Omega_{\kappa, \mu \pm 1}(\mathbf{n}) \\
& -\sqrt{2} \frac{\sqrt{\left(\kappa \pm \mu+\frac{1}{2}\right)\left(\kappa \pm \mu+\frac{3}{2}\right)}}{2 \kappa+1} \Omega_{-\kappa-1, \mu \pm 1}(\mathbf{n}) \\
& \mathbf{e}_{0} \cdot(\mathbf{n} \times \boldsymbol{\sigma}) \Omega_{\kappa \mu}(\mathbf{n})=i \frac{4 \mu \kappa}{4 \kappa^{2}-1} \Omega_{-\kappa \mu}(\mathbf{n})+i \frac{\sqrt{\left(\kappa+\frac{1}{2}\right)^{2}-\mu^{2}}}{|2 \kappa+1|} \Omega_{\kappa+1, \mu}(\mathbf{n}) \\
& -i \frac{\sqrt{\left(\kappa-\frac{1}{2}\right)^{2}-\mu^{2}}}{|2 \kappa-1|} \Omega_{\kappa-1, \mu}(\mathbf{n}) \\
& \mathbf{e}_{ \pm 1} \cdot(\mathbf{n} \times \boldsymbol{\sigma}) \Omega_{\kappa \mu}(\mathbf{n})=\mp i 2 \sqrt{2} \kappa \frac{\sqrt{\kappa^{2}-\left(\mu \pm \frac{1}{2}\right)^{2}}}{4 \kappa^{2}-1} \Omega_{-\kappa, \mu \pm 1}(\mathbf{n}) \\
& +i \frac{\sqrt{\left(\kappa \pm \mu+\frac{1}{2}\right)\left(\kappa \pm \mu+\frac{3}{2}\right)}}{\sqrt{2}(2 \kappa+1)} \Omega_{\kappa+1, \mu \pm 1}(\mathbf{n}) \\
& +i \frac{\sqrt{\left(\kappa \mp \mu-\frac{1}{2}\right)\left(\kappa \mp \mu-\frac{3}{2}\right)}}{\sqrt{2}(2 \kappa-1)} \Omega_{\kappa-1, \mu \pm 1}(\mathbf{n})
\end{aligned}
$$




\subsection{Differential relations of the first kind}

$$
\begin{aligned}
\mathbf{e}_{0} \cdot \hat{\mathbf{L}} \Omega_{\kappa \mu}(\mathbf{n})= & \frac{2 \mu(\kappa+1)}{2 \kappa+1} \Omega_{\kappa \mu}(\mathbf{n})+\frac{\sqrt{\left(\kappa+\frac{1}{2}\right)^{2}-\mu^{2}}}{|2 \kappa+1|} \Omega_{-\kappa-1, \mu}(\mathbf{n}) \\
\mathbf{e}_{ \pm 1} \cdot \hat{\mathbf{L}} \Omega_{\kappa \mu}(\mathbf{n})= & \mp \sqrt{2}(\kappa+1) \frac{\sqrt{\kappa^{2}-\left(\mu \pm \frac{1}{2}\right)^{2}}}{2 \kappa+1} \Omega_{\kappa, \mu \pm 1}(\mathbf{n}) \\
& +\frac{\sqrt{\left(\kappa \pm \mu+\frac{1}{2}\right)\left(\kappa \pm \mu+\frac{3}{2}\right)}}{\sqrt{2}(2 \kappa+1)} \Omega_{-\kappa-1, \mu \pm 1}(\mathbf{n})
\end{aligned}
$$

$\boldsymbol{\sigma} \cdot \hat{\mathbf{L}} \Omega_{\kappa \mu}(\mathbf{n})=-(\kappa+1) \Omega_{\kappa \mu}(\mathbf{n})$

$\mathbf{n} \cdot \hat{\mathbf{J}} \Omega_{\kappa \mu}(\mathbf{n})=-\frac{1}{2} \Omega_{-\kappa \mu}(\mathbf{n})$

$\mathbf{e}_{0} \cdot \hat{\mathbf{J}} \Omega_{\kappa \mu}(\mathbf{n})=\mu \Omega_{\kappa \mu}(\mathbf{n})$

$\mathbf{e}_{ \pm 1} \cdot \hat{\mathbf{J}} \Omega_{\kappa \mu}(\mathbf{n})=\mp \frac{1}{\sqrt{2}} \sqrt{\kappa^{2}-\left(\mu \pm \frac{1}{2}\right)^{2}} \Omega_{\kappa, \mu \pm 1}(\mathbf{n})$

$\boldsymbol{\sigma} \cdot \hat{\mathbf{J}} \Omega_{\kappa \mu}(\mathbf{n})=-\left(\kappa-\frac{1}{2}\right) \Omega_{\kappa \mu}(\mathbf{n})$

$\hat{\mathbf{L}}^{2} \Omega_{\kappa \mu}(\mathbf{n})=\kappa(\kappa+1) \Omega_{\kappa \mu}(\mathbf{n})$

$\hat{\mathbf{J}}^{2} \Omega_{\kappa \mu}(\mathbf{n})=\left(\kappa^{2}-\frac{1}{4}\right) \Omega_{\kappa \mu}(\mathbf{n})$

$\hat{\mathbf{L}} \cdot \hat{\mathbf{J}} \Omega_{\kappa \mu}(\mathbf{n})=\left(\kappa-\frac{1}{2}\right)(\kappa+1) \Omega_{\kappa \mu}(\mathbf{n})$

$$
\begin{aligned}
\mathbf{e}_{0} \cdot(\mathbf{n} \times \hat{\mathbf{L}}) \Omega_{\kappa \mu}(\mathbf{n})= & -i \frac{2 \mu(\kappa+1)}{4 \kappa^{2}-1} \Omega_{-\kappa \mu}(\mathbf{n})-i \kappa \frac{\sqrt{\left(\kappa+\frac{1}{2}\right)^{2}-\mu^{2}}}{|2 \kappa+1|} \Omega_{\kappa+1, \mu}(\mathbf{n}) \\
+ & i(\kappa+1) \frac{\sqrt{\left(\kappa-\frac{1}{2}\right)^{2}-\mu^{2}}}{|2 \kappa-1|} \Omega_{\kappa-1, \mu}(\mathbf{n}) \\
\mathbf{e}_{ \pm 1} \cdot(\mathbf{n} \times \hat{\mathbf{L}}) \Omega_{\kappa \mu}(\mathbf{n})= & \pm i \sqrt{2}(\kappa+1) \frac{\sqrt{\kappa^{2}-\left(\mu \pm \frac{1}{2}\right)^{2}}}{4 \kappa^{2}-1} \Omega_{-\kappa, \mu \pm 1}(\mathbf{n}) \\
& -i \kappa \frac{\sqrt{\left(\kappa \pm \mu+\frac{1}{2}\right)\left(\kappa \pm \mu+\frac{3}{2}\right)}}{\sqrt{2}(2 \kappa+1)} \Omega_{\kappa+1, \mu \pm 1}(\mathbf{n}) \\
& -i(\kappa+1) \frac{\sqrt{\left(\kappa \mp \mu-\frac{1}{2}\right)\left(\kappa \mp \mu-\frac{3}{2}\right)}}{\sqrt{2}(2 \kappa-1)} \Omega_{\kappa-1, \mu \pm 1}(\mathbf{n}) \\
\mathbf{e}_{0} \cdot(\hat{\mathbf{L}} \times \mathbf{n}) \Omega_{\kappa \mu}(\mathbf{n})= & i \frac{2 \mu(\kappa-1)}{4 \kappa^{2}-1} \Omega_{-\kappa \mu}(\mathbf{n})+i(\kappa+2) \frac{\sqrt{\left(\kappa+\frac{1}{2}\right)^{2}-\mu^{2}}}{|2 \kappa+1|} \Omega_{\kappa+1, \mu}(\mathbf{n}) \\
& -i(\kappa-1) \frac{\sqrt{\left(\kappa-\frac{1}{2}\right)^{2}-\mu^{2}}}{|2 \kappa-1|} \Omega_{\kappa-1, \mu}(\mathbf{n})
\end{aligned}
$$




$$
\begin{aligned}
\mathbf{e}_{ \pm 1} \cdot(\hat{\mathbf{L}} \times \mathbf{n}) \Omega_{\kappa \mu}(\mathbf{n})= & \mp i \sqrt{2}(\kappa-1) \frac{\sqrt{\kappa^{2}-\left(\mu \pm \frac{1}{2}\right)^{2}}}{4 \kappa^{2}-1} \Omega_{-\kappa, \mu \pm 1}(\mathbf{n}) \\
& +i(\kappa+2) \frac{\sqrt{\left(\kappa \pm \mu+\frac{1}{2}\right)\left(\kappa \pm \mu+\frac{3}{2}\right)}}{\sqrt{2}(2 \kappa+1)} \Omega_{\kappa+1, \mu \pm 1}(\mathbf{n}) \\
& +i(\kappa-1) \frac{\sqrt{\left(\kappa \mp \mu-\frac{1}{2}\right)\left(\kappa \mp \mu-\frac{3}{2}\right)}}{\sqrt{2}(2 \kappa-1)} \Omega_{\kappa-1, \mu \pm 1}(\mathbf{n})
\end{aligned}
$$

$\mathbf{n} \cdot(\boldsymbol{\sigma} \times \hat{\mathbf{L}}) \Omega_{\kappa \mu}(\mathbf{n})=i(\kappa+1) \Omega_{-\kappa \mu}(\mathbf{n})$

$$
\begin{aligned}
& \mathbf{e}_{0} \cdot(\boldsymbol{\sigma} \times \hat{\mathbf{L}}) \Omega_{\kappa \mu}(\mathbf{n})=i \operatorname{sgn}(\kappa) \sqrt{\left(\kappa+\frac{1}{2}\right)^{2}-\mu^{2}} \Omega_{-\kappa-1, \mu}(\mathbf{n}) \\
& \mathbf{e}_{ \pm 1} \cdot(\boldsymbol{\sigma} \times \hat{\mathbf{L}}) \Omega_{\kappa \mu}(\mathbf{n})=i \frac{1}{\sqrt{2}} \sqrt{\left(\kappa \pm \mu+\frac{1}{2}\right)\left(\kappa \pm \mu+\frac{3}{2}\right)} \Omega_{-\kappa-1, \mu \pm 1}(\mathbf{n}) \\
& (\boldsymbol{\sigma} \times \hat{\mathbf{L}}) \cdot \mathbf{n} \Omega_{\kappa \mu}(\mathbf{n})=i(\kappa-1) \Omega_{-\kappa \mu}(\mathbf{n})
\end{aligned}
$$

$$
\begin{aligned}
\mathbf{e}_{0} \cdot(\mathbf{n} \times \hat{\mathbf{J}}) \Omega_{\kappa \mu}(\mathbf{n})= & -i \frac{2 \mu}{4 \kappa^{2}-1} \Omega_{-\kappa \mu}(\mathbf{n})-i\left(\kappa-\frac{1}{2}\right) \frac{\sqrt{\left(\kappa+\frac{1}{2}\right)^{2}-\mu^{2}}}{|2 \kappa+1|} \Omega_{\kappa+1, \mu}(\mathbf{n}) \\
& +i\left(\kappa+\frac{1}{2}\right) \frac{\sqrt{\left(\kappa-\frac{1}{2}\right)^{2}-\mu^{2}}}{|2 \kappa-1|} \Omega_{\kappa-1, \mu}(\mathbf{n})
\end{aligned}
$$

$$
\mathbf{e}_{ \pm 1} \cdot(\mathbf{n} \times \hat{\mathbf{J}}) \Omega_{\kappa \mu}(\mathbf{n})= \pm i \sqrt{2} \frac{\sqrt{\kappa^{2}-\left(\mu \pm \frac{1}{2}\right)^{2}}}{4 \kappa^{2}-1} \Omega_{-\kappa, \mu \pm 1}(\mathbf{n})
$$

$$
\begin{aligned}
& -i\left(\kappa-\frac{1}{2}\right) \frac{\sqrt{\left(\kappa \pm \mu+\frac{1}{2}\right)\left(\kappa \pm \mu+\frac{3}{2}\right)}}{\sqrt{2}(2 \kappa+1)} \Omega_{\kappa+1, \mu \pm 1}(\mathbf{n}) \\
& -i\left(\kappa+\frac{1}{2}\right) \frac{\sqrt{\left(\kappa \mp \mu-\frac{1}{2}\right)\left(\kappa \mp \mu-\frac{3}{2}\right)}}{\sqrt{2}(2 \kappa-1)} \Omega_{\kappa-1, \mu \pm 1}(\mathbf{n})
\end{aligned}
$$

$$
\begin{aligned}
\mathbf{e}_{0} \cdot(\hat{\mathbf{J}} \times \mathbf{n}) \Omega_{\kappa \mu}(\mathbf{n})= & -i \frac{2 \mu}{4 \kappa^{2}-1} \Omega_{-\kappa \mu}(\mathbf{n})+i\left(\kappa+\frac{3}{2}\right) \frac{\sqrt{\left(\kappa+\frac{1}{2}\right)^{2}-\mu^{2}}}{|2 \kappa+1|} \Omega_{\kappa+1, \mu}(\mathbf{n}) \\
& -i\left(\kappa-\frac{3}{2}\right) \frac{\sqrt{\left(\kappa-\frac{1}{2}\right)^{2}-\mu^{2}}}{|2 \kappa-1|} \Omega_{\kappa-1, \mu}(\mathbf{n})
\end{aligned}
$$

$$
\begin{aligned}
\mathbf{e}_{ \pm 1} \cdot(\hat{\mathbf{J}} \times \mathbf{n}) \Omega_{\kappa \mu}(\mathbf{n})= & \pm i \sqrt{2} \frac{\sqrt{\kappa^{2}-\left(\mu \pm \frac{1}{2}\right)^{2}}}{4 \kappa^{2}-1} \Omega_{-\kappa, \mu \pm 1}(\mathbf{n}) \\
& +i\left(\kappa+\frac{3}{2}\right) \frac{\sqrt{\left(\kappa \pm \mu+\frac{1}{2}\right)\left(\kappa \pm \mu+\frac{3}{2}\right)}}{\sqrt{2}(2 \kappa+1)} \Omega_{\kappa+1, \mu \pm 1}(\mathbf{n}) \\
& +i\left(\kappa-\frac{3}{2}\right) \frac{\sqrt{\left(\kappa \mp \mu-\frac{1}{2}\right)\left(\kappa \mp \mu-\frac{3}{2}\right)}}{\sqrt{2}(2 \kappa-1)} \Omega_{\kappa-1, \mu \pm 1}(\mathbf{n})
\end{aligned}
$$

$\mathbf{n} \cdot(\boldsymbol{\sigma} \times \hat{\mathbf{J}}) \Omega_{\kappa \mu}(\mathbf{n})=i \kappa \Omega_{-\kappa \mu}(\mathbf{n})$ 


$$
\begin{aligned}
\mathbf{e}_{0} \cdot(\boldsymbol{\sigma} \times \hat{\mathbf{J}}) \Omega_{\kappa \mu}(\mathbf{n})=-i & \frac{2 \mu}{2 \kappa+1} \Omega_{\kappa \mu}(\mathbf{n})+i(2 \kappa-1) \frac{\sqrt{\left(\kappa+\frac{1}{2}\right)^{2}-\mu^{2}}}{|2 \kappa+1|} \Omega_{-\kappa-1, \mu}(\mathbf{n}) \\
\mathbf{e}_{ \pm 1} \cdot(\boldsymbol{\sigma} \times \hat{\mathbf{J}}) \Omega_{\kappa \mu}(\mathbf{n})= & \pm i \sqrt{2} \frac{\sqrt{\kappa^{2}-\left(\mu \pm \frac{1}{2}\right)^{2}}}{2 \kappa+1} \Omega_{\kappa, \mu \pm 1}(\mathbf{n}) \\
& +i(2 \kappa-1) \frac{\sqrt{\left(\kappa \pm \mu+\frac{1}{2}\right)\left(\kappa \pm \mu+\frac{3}{2}\right)}}{\sqrt{2}(2 \kappa+1)} \Omega_{-\kappa-1, \mu \pm 1}(\mathbf{n})
\end{aligned}
$$

$(\boldsymbol{\sigma} \times \hat{\mathbf{J}}) \cdot \mathbf{n} \Omega_{\kappa \mu}(\mathbf{n})=i(\kappa-2) \Omega_{-\kappa \mu}(\mathbf{n})$

$\mathbf{n} \cdot(\hat{\mathbf{J}} \times \boldsymbol{\sigma}) \Omega_{\kappa \mu}(\mathbf{n})=-i(\kappa+2) \Omega_{-\kappa \mu}(\mathbf{n})$

$\mathbf{e}_{0} \cdot(\hat{\mathbf{J}} \times \boldsymbol{\sigma}) \Omega_{\kappa \mu}(\mathbf{n})=-i \frac{2 \mu}{2 \kappa+1} \Omega_{\kappa \mu}(\mathbf{n})-i(2 \kappa+3) \frac{\sqrt{\left(\kappa+\frac{1}{2}\right)^{2}-\mu^{2}}}{|2 \kappa+1|} \Omega_{-\kappa-1, \mu}(\mathbf{n})$

$\mathbf{e}_{ \pm 1} \cdot(\hat{\mathbf{J}} \times \boldsymbol{\sigma}) \Omega_{\kappa \mu}(\mathbf{n})= \pm i \sqrt{2} \frac{\sqrt{\kappa^{2}-\left(\mu \pm \frac{1}{2}\right)^{2}}}{2 \kappa+1} \Omega_{\kappa, \mu \pm 1}(\mathbf{n})$

$$
-i(2 \kappa+3) \frac{\sqrt{\left(\kappa \pm \mu+\frac{1}{2}\right)\left(\kappa \pm \mu+\frac{3}{2}\right)}}{\sqrt{2}(2 \kappa+1)} \Omega_{-\kappa-1, \mu \pm 1}(\mathbf{n})
$$

$(\hat{\mathbf{J}} \times \boldsymbol{\sigma}) \cdot \mathbf{n} \Omega_{\kappa \mu}(\mathbf{n})=-i \kappa \Omega_{-\kappa \mu}(\mathbf{n})$

$\mathbf{n} \cdot(\hat{\mathbf{L}} \times \hat{\mathbf{J}}) \Omega_{\kappa \mu}(\mathbf{n})=-i \frac{1}{2}(\kappa+1) \Omega_{-\kappa \mu}(\mathbf{n})$

$\mathbf{e}_{0} \cdot(\hat{\mathbf{L}} \times \hat{\mathbf{J}}) \Omega_{\kappa \mu}(\mathbf{n})=i \frac{2 \mu(\kappa+1)}{2 \kappa+1} \Omega_{\kappa \mu}(\mathbf{n})-i\left(\kappa-\frac{1}{2}\right) \frac{\sqrt{\left(\kappa+\frac{1}{2}\right)^{2}-\mu^{2}}}{|2 \kappa+1|} \Omega_{-\kappa-1, \mu}(\mathbf{n})$

$\mathbf{e}_{ \pm 1} \cdot(\hat{\mathbf{L}} \times \hat{\mathbf{J}}) \Omega_{\kappa \mu}(\mathbf{n})=\mp i \sqrt{2}(\kappa+1) \frac{\sqrt{\kappa^{2}-\left(\mu \pm \frac{1}{2}\right)^{2}}}{2 \kappa+1} \Omega_{\kappa, \mu \pm 1}(\mathbf{n})$

$$
-i\left(\kappa-\frac{1}{2}\right) \frac{\sqrt{\left(\kappa \pm \mu+\frac{1}{2}\right)\left(\kappa \pm \mu+\frac{3}{2}\right)}}{\sqrt{2}(2 \kappa+1)} \Omega_{-\kappa-1, \mu \pm 1}(\mathbf{n})
$$

$(\hat{\mathbf{L}} \times \hat{\mathbf{J}}) \cdot \mathbf{n} \Omega_{\kappa \mu}(\mathbf{n})=-i \frac{1}{2}(\kappa-1) \Omega_{-\kappa \mu}(\mathbf{n})$

$(\hat{\mathbf{L}} \times \hat{\mathbf{J}}) \cdot \boldsymbol{\sigma} \Omega_{\kappa \mu}(\mathbf{n})=-i 2(\kappa+1) \Omega_{\kappa \mu}(\mathbf{n})$

$\mathbf{n} \cdot(\hat{\mathbf{J}} \times \hat{\mathbf{L}}) \Omega_{\kappa \mu}(\mathbf{n})=i \frac{1}{2}(\kappa+1) \Omega_{-\kappa \mu}(\mathbf{n})$

$\mathbf{e}_{0} \cdot(\hat{\mathbf{J}} \times \hat{\mathbf{L}}) \Omega_{\kappa \mu}(\mathbf{n})=i \frac{2 \mu(\kappa+1)}{2 \kappa+1} \Omega_{\kappa \mu}(\mathbf{n})+i\left(\kappa+\frac{3}{2}\right) \frac{\sqrt{\left(\kappa+\frac{1}{2}\right)^{2}-\mu^{2}}}{|2 \kappa+1|} \Omega_{-\kappa-1, \mu}(\mathbf{n})$ 


$$
\begin{aligned}
& \mathbf{e}_{ \pm 1} \cdot(\hat{\mathbf{J}} \times \hat{\mathbf{L}}) \Omega_{\kappa \mu}(\mathbf{n})=\mp i \sqrt{2}(\kappa+1) \frac{\sqrt{\kappa^{2}-\left(\mu \pm \frac{1}{2}\right)^{2}}}{2 \kappa+1} \Omega_{\kappa, \mu \pm 1}(\mathbf{n}) \\
& +i\left(\kappa+\frac{3}{2}\right) \frac{\sqrt{\left(\kappa \pm \mu+\frac{1}{2}\right)\left(\kappa \pm \mu+\frac{3}{2}\right)}}{\sqrt{2}(2 \kappa+1)} \Omega_{-\kappa-1, \mu \pm 1}(\mathbf{n}) \\
& \boldsymbol{\sigma} \cdot(\hat{\mathbf{J}} \times \hat{\mathbf{L}}) \Omega_{\kappa \mu}(\mathbf{n})=-i 2(\kappa+1) \Omega_{\kappa \mu}(\mathbf{n}) \\
& (\hat{\mathbf{J}} \times \hat{\mathbf{L}}) \cdot \mathbf{n} \Omega_{\kappa \mu}(\mathbf{n})=i \frac{1}{2}(\kappa-1) \Omega_{-\kappa \mu}(\mathbf{n}) \\
& \hat{\mathbf{L}} \cdot(\mathbf{n} \times \hat{\mathbf{J}}) \Omega_{\kappa \mu}(\mathbf{n})=i \frac{1}{2}(\kappa-1) \Omega_{-\kappa \mu}(\mathbf{n}) \\
& \hat{\mathbf{J}} \cdot(\mathbf{n} \times \hat{\mathbf{L}}) \Omega_{\kappa \mu}(\mathbf{n})=-i \frac{1}{2}(\kappa+1) \Omega_{-\kappa \mu}(\mathbf{n}) \\
& \boldsymbol{\sigma} \cdot(\hat{\mathbf{J}} \times \boldsymbol{\sigma}) \Omega_{\kappa \mu}(\mathbf{n})=i(2 \kappa+5) \Omega_{\kappa \mu}(\mathbf{n}) \\
& \hat{\mathbf{J}} \cdot(\mathbf{n} \times \hat{\mathbf{J}}) \Omega_{\kappa \mu}(\mathbf{n})=-i \frac{1}{2} \Omega_{-\kappa \mu}(\mathbf{n}) \\
& \hat{\mathbf{J}} \cdot(\boldsymbol{\sigma} \times \hat{\mathbf{J}}) \Omega_{\kappa \mu}(\mathbf{n})=-i\left(\kappa-\frac{1}{2}\right) \Omega_{\kappa \mu}(\mathbf{n}) \\
& \hat{\mathbf{J}} \cdot(\hat{\mathbf{L}} \times \hat{\mathbf{J}}) \Omega_{\kappa \mu}(\mathbf{n})=i\left(\kappa-\frac{1}{2}\right)(\kappa+1) \Omega_{\kappa \mu}(\mathbf{n}) \\
& (\hat{\mathbf{L}} \times \hat{\mathbf{J}}) \cdot \hat{\mathbf{L}} \Omega_{\kappa \mu}(\mathbf{n})=i\left(\kappa+\frac{1}{2}\right)(\kappa+1) \Omega_{\kappa \mu}(\mathbf{n})
\end{aligned}
$$

\subsection{Differential relations of the second kind}

$$
\begin{aligned}
\mathbf{n} \cdot \nabla F(r) \Omega_{\kappa \mu}(\mathbf{n})= & \frac{\mathrm{d} F(r)}{\mathrm{d} r} \Omega_{\kappa \mu}(\mathbf{n}) \\
\boldsymbol{\nabla} \cdot \mathbf{n} F(r) \Omega_{\kappa \mu}(\mathbf{n})=\left(\frac{\partial}{\partial r}+\frac{2}{r}\right) F(r) \Omega_{\kappa \mu}(\mathbf{n}) & \\
\mathbf{e}_{0} \cdot \boldsymbol{\nabla} F(r) \Omega_{\kappa \mu}(\mathbf{n})= & -\frac{2 \mu}{4 \kappa^{2}-1}\left(\frac{\partial}{\partial r}+\frac{\kappa+1}{r}\right) F(r) \Omega_{-\kappa \mu}(\mathbf{n}) \\
+ & \frac{\sqrt{\left(\kappa+\frac{1}{2}\right)^{2}-\mu^{2}}}{|2 \kappa+1|}\left(\frac{\partial}{\partial r}-\frac{\kappa}{r}\right) F(r) \Omega_{\kappa+1, \mu}(\mathbf{n}) \\
& +\frac{\sqrt{\left(\kappa-\frac{1}{2}\right)^{2}-\mu^{2}}}{|2 \kappa-1|}\left(\frac{\partial}{\partial r}+\frac{\kappa+1}{r}\right) F(r) \Omega_{\kappa-1, \mu}(\mathbf{n}) \\
\mathbf{e}_{ \pm 1} \cdot \boldsymbol{\nabla} F(r) \Omega_{\kappa \mu}(\mathbf{n})= & \pm \sqrt{2} \frac{\sqrt{\kappa^{2}-\left(\mu \pm \frac{1}{2}\right)^{2}}}{4 \kappa^{2}-1}\left(\frac{\partial}{\partial r}+\frac{\kappa+1}{r}\right) F(r) \Omega_{-\kappa, \mu \pm 1}(\mathbf{n}) \\
& +\frac{\sqrt{\left(\kappa \pm \mu+\frac{1}{2}\right)\left(\kappa \pm \mu+\frac{3}{2}\right)}}{\sqrt{2}(2 \kappa+1)}\left(\frac{\partial}{\partial r}-\frac{\kappa}{r}\right) F(r) \Omega_{\kappa+1, \mu \pm 1}(\mathbf{n}) \\
& -\frac{\sqrt{\left(\kappa \mp \mu-\frac{1}{2}\right)\left(\kappa \mp \mu-\frac{3}{2}\right)}}{\sqrt{2}(2 \kappa-1)}\left(\frac{\partial}{\partial r}+\frac{\kappa+1}{r}\right) F(r) \Omega_{\kappa-1, \mu \pm 1}(\mathbf{n})
\end{aligned}
$$




$$
\begin{aligned}
& \boldsymbol{\sigma} \cdot \boldsymbol{\nabla} F(r) \Omega_{\kappa \mu}(\mathbf{n})=-\left(\frac{\partial}{\partial r}+\frac{\kappa+1}{r}\right) F(r) \Omega_{-\kappa \mu}(\mathbf{n}) \\
& \hat{\mathbf{J}} \cdot \nabla F(r) \Omega_{\kappa \mu}(\mathbf{n})=-\frac{1}{2}\left(\frac{\partial}{\partial r}+\frac{\kappa+1}{r}\right) F(r) \Omega_{-\kappa \mu}(\mathbf{n}) \\
& \boldsymbol{\nabla} \cdot \hat{\mathbf{J}} F(r) \Omega_{\kappa \mu}(\mathbf{n})=-\frac{1}{2}\left(\frac{\partial}{\partial r}+\frac{\kappa+1}{r}\right) F(r) \Omega_{-\kappa \mu}(\mathbf{n}) \\
& \nabla^{2} F(r) \Omega_{\kappa \mu}(\mathbf{n})=\frac{1}{r}\left(\frac{\partial^{2}}{\partial r^{2}}-\frac{\kappa(\kappa+1)}{r^{2}}\right) r F(r) \Omega_{\kappa \mu}(\mathbf{n}) \\
& \mathbf{e}_{0} \cdot(\boldsymbol{\sigma} \times \boldsymbol{\nabla}) F(r) \Omega_{\kappa \mu}(\mathbf{n})=-i \frac{4 \mu \kappa}{4 \kappa^{2}-1}\left(\frac{\partial}{\partial r}+\frac{\kappa+1}{r}\right) F(r) \Omega_{-\kappa \mu}(\mathbf{n}) \\
& -i \frac{\sqrt{\left(\kappa+\frac{1}{2}\right)^{2}-\mu^{2}}}{|2 \kappa+1|}\left(\frac{\partial}{\partial r}-\frac{\kappa}{r}\right) F(r) \Omega_{\kappa+1, \mu}(\mathbf{n}) \\
& +i \frac{\sqrt{\left(\kappa-\frac{1}{2}\right)^{2}-\mu^{2}}}{|2 \kappa-1|}\left(\frac{\partial}{\partial r}+\frac{\kappa+1}{r}\right) F(r) \Omega_{\kappa-1, \mu}(\mathbf{n}) \\
& \mathbf{e}_{ \pm 1} \cdot(\boldsymbol{\sigma} \times \boldsymbol{\nabla}) F(r) \Omega_{\kappa \mu}(\mathbf{n})= \pm i 2 \sqrt{2} \kappa \frac{\sqrt{\kappa^{2}-\left(\mu \pm \frac{1}{2}\right)^{2}}}{4 \kappa^{2}-1}\left(\frac{\partial}{\partial r}+\frac{\kappa+1}{r}\right) F(r) \Omega_{-\kappa, \mu \pm 1}(\mathbf{n}) \\
& -i \frac{\sqrt{\left(\kappa \pm \mu+\frac{1}{2}\right)\left(\kappa \pm \mu+\frac{3}{2}\right)}}{\sqrt{2}(2 \kappa+1)}\left(\frac{\partial}{\partial r}-\frac{\kappa}{r}\right) F(r) \Omega_{\kappa+1, \mu \pm 1}(\mathbf{n}) \\
& -i \frac{\sqrt{\left(\kappa \mp \mu-\frac{1}{2}\right)\left(\kappa \mp \mu-\frac{3}{2}\right)}}{\sqrt{2}(2 \kappa-1)}\left(\frac{\partial}{\partial r}+\frac{\kappa+1}{r}\right) F(r) \Omega_{\kappa-1, \mu \pm 1}(\mathbf{n}) \\
& \hat{\mathbf{J}} \cdot(\boldsymbol{\sigma} \times \boldsymbol{\nabla}) F(r) \Omega_{\kappa \mu}(\mathbf{n})=-i \kappa\left(\frac{\partial}{\partial r}+\frac{\kappa+1}{r}\right) F(r) \Omega_{-\kappa \mu}(\mathbf{n}) \\
& \mathbf{n} \cdot(\hat{\mathbf{L}} \times \boldsymbol{\nabla}) F(r) \Omega_{\kappa \mu}(\mathbf{n})=i\left(2 \frac{\partial}{\partial r}-\frac{\kappa(\kappa+1)}{r}\right) F(r) \Omega_{\kappa \mu}(\mathbf{n}) \\
& \mathbf{e}_{0} \cdot(\hat{\mathbf{L}} \times \nabla) F(r) \Omega_{\kappa \mu}(\mathbf{n})=i \frac{2 \mu(\kappa-1)}{4 \kappa^{2}-1}\left(\frac{\partial}{\partial r}+\frac{\kappa+1}{r}\right) F(r) \Omega_{-\kappa \mu}(\mathbf{n}) \\
& +i(\kappa+2) \frac{\sqrt{\left(\kappa+\frac{1}{2}\right)^{2}-\mu^{2}}}{|2 \kappa+1|}\left(\frac{\partial}{\partial r}-\frac{\kappa}{r}\right) F(r) \Omega_{\kappa+1, \mu}(\mathbf{n}) \\
& -i(\kappa-1) \frac{\sqrt{\left(\kappa-\frac{1}{2}\right)^{2}-\mu^{2}}}{|2 \kappa-1|}\left(\frac{\partial}{\partial r}+\frac{\kappa+1}{r}\right) F(r) \Omega_{\kappa-1, \mu}(\mathbf{n})
\end{aligned}
$$




$$
\begin{aligned}
& \mathbf{e}_{ \pm 1} \cdot(\hat{\mathbf{L}} \times \nabla) F(r) \Omega_{\kappa \mu}(\mathbf{n})=\mp i \sqrt{2}(\kappa-1) \frac{\sqrt{\kappa^{2}-\left(\mu \pm \frac{1}{2}\right)^{2}}}{4 \kappa^{2}-1}\left(\frac{\partial}{\partial r}+\frac{\kappa+1}{r}\right) F(r) \Omega_{-\kappa, \mu \pm 1}(\mathbf{n}) \\
& +i(\kappa+2) \frac{\sqrt{\left(\kappa \pm \mu+\frac{1}{2}\right)\left(\kappa \pm \mu+\frac{3}{2}\right)}}{\sqrt{2}(2 \kappa+1)}\left(\frac{\partial}{\partial r}-\frac{\kappa}{r}\right) F(r) \Omega_{\kappa+1, \mu \pm 1}(\mathbf{n}) \\
& +i(\kappa-1) \frac{\sqrt{\left(\kappa \mp \mu-\frac{1}{2}\right)\left(\kappa \mp \mu-\frac{3}{2}\right)}}{\sqrt{2}(2 \kappa-1)}\left(\frac{\partial}{\partial r}+\frac{\kappa+1}{r}\right) F(r) \Omega_{\kappa-1, \mu \pm 1}(\mathbf{n}) \\
& \boldsymbol{\sigma} \cdot(\hat{\mathbf{L}} \times \boldsymbol{\nabla}) F(r) \Omega_{\kappa \mu}(\mathbf{n})=i(\kappa-1)\left(\frac{\partial}{\partial r}+\frac{\kappa+1}{r}\right) F(r) \Omega_{-\kappa \mu}(\mathbf{n}) \\
& \hat{\mathbf{J}} \cdot(\hat{\mathbf{L}} \times \boldsymbol{\nabla}) F(r) \Omega_{\kappa \mu}(\mathbf{n})=i \frac{1}{2}(\kappa-1)\left(\frac{\partial}{\partial r}+\frac{\kappa+1}{r}\right) F(r) \Omega_{-\kappa \mu}(\mathbf{n}) \\
& \mathbf{e}_{0} \cdot(\boldsymbol{\nabla} \times \hat{\mathbf{L}}) F(r) \Omega_{\kappa \mu}(\mathbf{n})=-i \frac{2 \mu(\kappa+1)}{4 \kappa^{2}-1}\left(\frac{\partial}{\partial r}+\frac{\kappa+1}{r}\right) F(r) \Omega_{-\kappa \mu}(\mathbf{n}) \\
& -i \kappa \frac{\sqrt{\left(\kappa+\frac{1}{2}\right)^{2}-\mu^{2}}}{|2 \kappa+1|}\left(\frac{\partial}{\partial r}-\frac{\kappa}{r}\right) F(r) \Omega_{\kappa+1, \mu}(\mathbf{n}) \\
& +i(\kappa+1) \frac{\sqrt{\left(\kappa-\frac{1}{2}\right)^{2}-\mu^{2}}}{|2 \kappa-1|}\left(\frac{\partial}{\partial r}+\frac{\kappa+1}{r}\right) F(r) \Omega_{\kappa-1, \mu}(\mathbf{n}) \\
& \mathbf{e}_{ \pm 1} \cdot(\boldsymbol{\nabla} \times \hat{\mathbf{L}}) F(r) \Omega_{\kappa \mu}(\mathbf{n})= \pm i \sqrt{2}(\kappa+1) \frac{\sqrt{\kappa^{2}-\left(\mu \pm \frac{1}{2}\right)^{2}}}{4 \kappa^{2}-1}\left(\frac{\partial}{\partial r}+\frac{\kappa+1}{r}\right) F(r) \Omega_{-\kappa, \mu \pm 1}(\mathbf{n}) \\
& -i \kappa \frac{\sqrt{\left(\kappa \pm \mu+\frac{1}{2}\right)\left(\kappa \pm \mu+\frac{3}{2}\right)}}{\sqrt{2}(2 \kappa+1)}\left(\frac{\partial}{\partial r}-\frac{\kappa}{r}\right) F(r) \Omega_{\kappa+1, \mu \pm 1}(\mathbf{n}) \\
& -i(\kappa+1) \frac{\sqrt{\left(\kappa \mp \mu-\frac{1}{2}\right)\left(\kappa \mp \mu-\frac{3}{2}\right)}}{\sqrt{2}(2 \kappa-1)}\left(\frac{\partial}{\partial r}+\frac{\kappa+1}{r}\right) F(r) \Omega_{\kappa-1, \mu \pm 1}(\mathbf{n})
\end{aligned}
$$




$$
\begin{aligned}
& \mathbf{e}_{0} \cdot(\hat{\mathbf{J}} \times \boldsymbol{\nabla}) F(r) \Omega_{\kappa \mu}(\mathbf{n})=-i \frac{2 \mu}{4 \kappa^{2}-1}\left(\frac{\partial}{\partial r}+\frac{\kappa+1}{r}\right) F(r) \Omega_{-\kappa \mu}(\mathbf{n}) \\
& +i\left(\kappa+\frac{3}{2}\right) \frac{\sqrt{\left(\kappa+\frac{1}{2}\right)^{2}-\mu^{2}}}{|2 \kappa+1|}\left(\frac{\partial}{\partial r}-\frac{\kappa}{r}\right) F(r) \Omega_{\kappa+1, \mu}(\mathbf{n}) \\
& -i\left(\kappa-\frac{3}{2}\right) \frac{\sqrt{\left(\kappa-\frac{1}{2}\right)^{2}-\mu^{2}}}{|2 \kappa-1|}\left(\frac{\partial}{\partial r}+\frac{\kappa+1}{r}\right) F(r) \Omega_{\kappa-1, \mu}(\mathbf{n}) \\
& \mathbf{e}_{ \pm 1} \cdot(\hat{\mathbf{J}} \times \nabla) F(r) \Omega_{\kappa \mu}(\mathbf{n})= \pm i \sqrt{2} \frac{\sqrt{\kappa^{2}-\left(\mu \pm \frac{1}{2}\right)^{2}}}{4 \kappa^{2}-1}\left(\frac{\partial}{\partial r}+\frac{\kappa+1}{r}\right) F(r) \Omega_{-\kappa, \mu \pm 1}(\mathbf{n}) \\
& +i\left(\kappa+\frac{3}{2}\right) \frac{\sqrt{\left(\kappa \pm \mu+\frac{1}{2}\right)\left(\kappa \pm \mu+\frac{3}{2}\right)}}{\sqrt{2}(2 \kappa+1)}\left(\frac{\partial}{\partial r}-\frac{\kappa}{r}\right) F(r) \Omega_{\kappa+1, \mu \pm 1}(\mathbf{n}) \\
& +i\left(\kappa-\frac{3}{2}\right) \frac{\sqrt{\left(\kappa \mp \mu-\frac{1}{2}\right)\left(\kappa \mp \mu-\frac{3}{2}\right)}}{\sqrt{2}(2 \kappa-1)}\left(\frac{\partial}{\partial r}+\frac{\kappa+1}{r}\right) F(r) \Omega_{\kappa-1, \mu \pm 1}(\mathbf{n}) \\
& \boldsymbol{\sigma} \cdot(\hat{\mathbf{J}} \times \boldsymbol{\nabla}) F(r) \Omega_{\kappa \mu}(\mathbf{n})=i(\kappa-2)\left(\frac{\partial}{\partial r}+\frac{\kappa+1}{r}\right) F(r) \Omega_{-\kappa \mu}(\mathbf{n}) \\
& \hat{\mathbf{L}} \cdot(\hat{\mathbf{J}} \times \boldsymbol{\nabla}) F(r) \Omega_{\kappa \mu}(\mathbf{n})=-i \frac{1}{2}(\kappa-1)\left(\frac{\partial}{\partial r}+\frac{\kappa+1}{r}\right) F(r) \Omega_{-\kappa \mu}(\mathbf{n}) \\
& \mathbf{e}_{0} \cdot(\boldsymbol{\nabla} \times \hat{\mathbf{J}}) F(r) \Omega_{\kappa \mu}(\mathbf{n})=-i \frac{2 \mu}{4 \kappa^{2}-1}\left(\frac{\partial}{\partial r}+\frac{\kappa+1}{r}\right) F(r) \Omega_{-\kappa \mu}(\mathbf{n}) \\
& -i\left(\kappa-\frac{1}{2}\right) \frac{\sqrt{\left(\kappa+\frac{1}{2}\right)^{2}-\mu^{2}}}{|2 \kappa+1|}\left(\frac{\partial}{\partial r}-\frac{\kappa}{r}\right) F(r) \Omega_{\kappa+1, \mu}(\mathbf{n}) \\
& +i\left(\kappa+\frac{1}{2}\right) \frac{\sqrt{\left(\kappa-\frac{1}{2}\right)^{2}-\mu^{2}}}{|2 \kappa-1|}\left(\frac{\partial}{\partial r}+\frac{\kappa+1}{r}\right) F(r) \Omega_{\kappa-1, \mu}(\mathbf{n}) \\
& \mathbf{e}_{ \pm 1} \cdot(\boldsymbol{\nabla} \times \hat{\mathbf{J}}) F(r) \Omega_{\kappa \mu}(\mathbf{n})= \pm i \sqrt{2} \frac{\sqrt{\kappa^{2}-\left(\mu \pm \frac{1}{2}\right)^{2}}}{4 \kappa^{2}-1}\left(\frac{\partial}{\partial r}+\frac{\kappa+1}{r}\right) F(r) \Omega_{-\kappa, \mu \pm 1}(\mathbf{n}) \\
& -i\left(\kappa-\frac{1}{2}\right) \frac{\sqrt{\left(\kappa \pm \mu+\frac{1}{2}\right)\left(\kappa \pm \mu+\frac{3}{2}\right)}}{\sqrt{2}(2 \kappa+1)}\left(\frac{\partial}{\partial r}-\frac{\kappa}{r}\right) F(r) \Omega_{\kappa+1, \mu \pm 1}(\mathbf{n}) \\
& -i\left(\kappa+\frac{1}{2}\right) \frac{\sqrt{\left(\kappa \mp \mu-\frac{1}{2}\right)\left(\kappa \mp \mu-\frac{3}{2}\right)}}{\sqrt{2}(2 \kappa-1)}\left(\frac{\partial}{\partial r}+\frac{\kappa+1}{r}\right) F(r) \Omega_{\kappa-1, \mu \pm 1}(\mathbf{n}) \\
& \boldsymbol{\sigma} \cdot(\boldsymbol{\nabla} \times \hat{\mathbf{J}}) F(r) \Omega_{\kappa \mu}(\mathbf{n})=-i \kappa\left(\frac{\partial}{\partial r}+\frac{\kappa+1}{r}\right) F(r) \Omega_{-\kappa \mu}(\mathbf{n}) \\
& \hat{\mathbf{L}} \cdot(\boldsymbol{\nabla} \times \hat{\mathbf{J}}) F(r) \Omega_{\kappa \mu}(\mathbf{n})=i \frac{1}{2}(\kappa-1)\left(\frac{\partial}{\partial r}+\frac{\kappa+1}{r}\right) F(r) \Omega_{-\kappa \mu}(\mathbf{n})
\end{aligned}
$$




$$
\begin{aligned}
& \hat{\mathbf{J}} \cdot(\boldsymbol{\nabla} \times \hat{\mathbf{J}}) F(r) \Omega_{\kappa \mu}(\mathbf{n})=-i \frac{1}{2}\left(\frac{\partial}{\partial r}+\frac{\kappa+1}{r}\right) F(r) \Omega_{-\kappa \mu}(\mathbf{n}) \\
& (\boldsymbol{\nabla} \times \hat{\mathbf{J}}) \cdot \mathbf{n} F(r) \Omega_{\kappa \mu}(\mathbf{n})=i\left(2 \frac{\partial}{\partial r}+\frac{2 \kappa^{2}+\kappa+7}{2 r}\right) F(r) \Omega_{\kappa \mu}(\mathbf{n}) \\
& (\boldsymbol{\nabla} \times \hat{\mathbf{J}}) \cdot \boldsymbol{\sigma} F(r) \Omega_{\kappa \mu}(\mathbf{n})=-i(\kappa+2)\left(\frac{\partial}{\partial r}+\frac{\kappa+1}{r}\right) F(r) \Omega_{-\kappa \mu}(\mathbf{n}) \\
& (\boldsymbol{\nabla} \times \hat{\mathbf{J}}) \cdot \hat{\mathbf{L}} F(r) \Omega_{\kappa \mu}(\mathbf{n})=i \frac{1}{2}(\kappa+1)\left(\frac{\partial}{\partial r}+\frac{\kappa+1}{r}\right) F(r) \Omega_{-\kappa \mu}(\mathbf{n}) \\
& \boldsymbol{\nabla} \cdot(\hat{\mathbf{L}} \times \boldsymbol{\nabla}) F(r) \Omega_{\kappa \mu}(\mathbf{n})=i \frac{2}{r}\left(\frac{\partial^{2}}{\partial r^{2}}-\frac{\kappa(\kappa+1)}{r^{2}}\right) r F(r) \Omega_{\kappa \mu}(\mathbf{n}) \\
& \nabla \cdot(\hat{\mathbf{J}} \times \boldsymbol{\nabla}) F(r) \Omega_{\kappa \mu}(\mathbf{n})=i \frac{2}{r}\left(\frac{\partial^{2}}{\partial r^{2}}-\frac{\kappa(\kappa+1)}{r^{2}}\right) r F(r) \Omega_{\kappa \mu}(\mathbf{n})
\end{aligned}
$$

\section{Acknowledgments}

The author acknowledges discussions with Sebastian Bielski, Justyna Kunicka, and Krzysztof Mielewczyk.

\section{References}

[1] A. I. Akhiezer, V. B. Berestetskii, Quantum Electrodynamics, 2nd ed., GIFML, Moscow, 1959 (in Russian).

[2] M. E. Rose, Relativistic Electron Theory, Wiley, New York, 1961.

[3] J. D. Bjorken, S. D. Drell, Relativistic Quantum Mechanics, McGraw-Hill, New York, 1964.

[4] W. Greiner, Relativistic Quantum Theory. Wave Equations, Springer, Berlin, 1990.

[5] B. Thaller, The Dirac Equation, Springer, Berlin, 1992.

[6] P. Pyykkö, Relativistic Theory of Atoms and Molecules. A Bibliography 1916-1985, Springer, Berlin, 1986.

[7] P. Pyykkö, Relativistic Theory of Atoms and Molecules. II. A Bibliography 1986-1992, Springer, Berlin, 1993.

[8] P. Pyykkö, Relativistic Theory of Atoms and Molecules. III. A Bibliography 1993-1999, Springer, Berlin, 2000.

[9] Database RTAM at http://www.csc.fi/rtam/.

[10] M. E. Rose, Elementary Theory of Angular Momentum, Wiley, New York, 1957.

[11] A. R. Edmonds, Angular Momentum in Quantum Mechanics, 2nd ed., Princeton University Press, Princeton, NJ, 1960.

[12] L. C. Biedenharn, J. D. Louck, Angular Momentum in Quantum Physics, Addison-Wesley, Reading, Mass., 1981.

[13] J. D. Louck, in: Atomic, Molecular, and Optical Physics Handbook, ed. G. W. F. Drake, American Institute of Physics, Woodbury, NY, 1996, p. 6. 
[14] D. A. Varshalovich, A. N. Moskalev, V. K. Khersonskii, Quantum Theory of Angular Momentum. Irreducible Tensors, Spherical Harmonics, Vector Coupling Coefficients, 3nj-symbols, Nauka, Leningrad, 1975 (in Russian), World Scientific, Singapore, 1988 (in English).

[15] A. Bechler, J. Phys. A 26 (1993) 6039.

[16] A. Rutkowski, R. Kozłowski, J. Phys. B 30 (1997) 1437.

[17] R. Szmytkowski, J. Phys. B 35 (2002) 1379.

[18] R. Szmytkowski, K. Mielewczyk, J. Phys. B 37 (2004) 3961.

[19] K. Mielewczyk, R. Szmytkowski, Phys. Rev. A 73 (2006) 022511, 039908(E).

[20] R. Szmytkowski, J. Phys. A 38 (2005) 8993.

[21] E. U. Condon, G. H. Shortley, The Theory of Atomic Spectra, Cambridge University Press, Cambridge, 1935. 